\title{
Forecasted Affect Towards Automated and Warning Safety Features
}

Brittany E. Noah

Georgia Institute of Technology

Atlanta, GA 30306, USA

brittany.noah@gatech.edu

Thomas M. Gable

Georgia Institute of Technology

Atlanta, GA 30306, USA

thomas.gable@gatech.edu

\section{Jonathan H. Schuett}

Georgia Institute of Technology

Atlanta, GA 30306, USA

jschuett6@gatech.edu

\section{Bruce N. Walker}

Georgia Institute of Technology

Atlanta, GA 30306, USA

bruce.walker@gatech.edu

Permission to make digital or hard copies of part or all of this work for personal or classroom use is granted without fee provided that copies are not made or distributed for profit or commercial advantage and that copies bear this notice and the full citation on the first page. Copyrights for third-party components of this work must be honored. For all other uses, contact the Owner/Author.

uses, contact the Owner/Author.
Copyright is held by the owner/author(s).

Automotive'UI 16 Adjunct, October 24-26, 2016, Ann Arbor, MI, USA

Automotive'UI 16 Adjunct, Octob

http://dx.doi.org/10.1145/3004323.3004337

\begin{abstract}
Driver perception of automated and warning safety features influence if, and how, drivers will use them. Affective forecasting from participants showed that there is higher predicted negative affect towards automated safety features, such as automated lane keeping, than towards warning safety features such as lane departure warnings. Additionally, driver confidence and technology experience were correlated with affective forecasting results. Future behavioral studies will determine if the affective forecasting results are accurate or more extreme than emotions felt at the time of rating.
\end{abstract}

\section{Author Keywords}

Automated driving; affective forecasting; confidence; PANAS.

\section{ACM Classification Keywords}

H.1.2 [User/Machine Systems]: Human factors; H.5.2 [Information Interfaces And Presentation (e.g., HCI)]: Evaluation/methodology, Graphical user interfaces (GUI), Screen design (e.g., text, graphics, color), User-centered design. 


\section{Introduction}

Automated driving

Level of automation is determined by the amount of input a human operator has into a system. Higher levels of automation indicate lower levels of human input; whereas, lower levels of automation indicate higher levels of human input required [7,3] As the level of automation in a system increases, decreasing required human input, the human moves to a supervisory rather than direct control modes [7]. In order to successfully integrate systems with higher levels of automation into existing technologies, such as automated features in vehicles, drivers must be able to understand how the technology works and the limitations of the technology [7].

\section{Safety features}

There are differing levels of automation available in new vehicles purchased today. Some safety features such as forward collision alerts, lane departure warnings, and rear parking assist are warnings that provide the driver with information but do not act on their behalf. Other safety features such as forward collision avoidance (emergency braking), automated lane keeping, adaptive cruise control, and automatic (assistive) parking have higher levels of automation, which act on the driver's behalf to avoid accidents.

\section{Affect}

Affect is the emotional experience of an individual at a given time. It is more transient than trait levels of positivity and negativity and changes frequently with context. Positive affect, or positive emotional state, and negative affect, or negative emotional state, are not mutually exclusive. Both of these emotions can be experienced toward a given scenario to some extent as both are experienced on a spectrum [8]. The Positive and Negative Affect Schedule was developed to determine how much positive affect (PA) and negative affect (NA) a person experienced during a given time from the immediate past to the further past [8].

\section{Affective forecasting}

Affective forecasting is the act of predicting a future emotional state [4]. While the PANAS scale was initially developed to serve as a self-report measure for past or present emotional states, affective forecasting leverages the same scale with instructions for participants to imagine what they would feel like in a given scenario [1]. Some studies have determined that affective forecasting is not accurate $[1,2,6]$. In a study where people did a multitasking homework task Calderwood and colleagues [1] found that students predicted much higher levels of NA when asked how they would feel if they were not allowed to use technology while completing homework. While researchers predicted students would also have increased PA if allowed to multitask while completing homework, the results did not show a significant difference between PA predictions across the multitasking allowed and removed conditions.

Present Study

This is a preliminary review of a larger data set that was collected, in part, to determine factors that may affect drivers' perceptions of the usefulness of automated and warning safety systems. Ongoing additional work aims to determine and weigh these factors to predict which demographic variables, such as age, and education, combined with driving experience in different environments, influence the usefulness of different safety features within a driving environment. 


\begin{tabular}{|c|c|}
\hline Age ranges & Frequency \\
\hline $18-19$ & 5 \\
\hline $20-24$ & 25 \\
\hline $25-29$ & 49 \\
\hline $30-34$ & 47 \\
\hline $35-39$ & 28 \\
\hline $40-44$ & 20 \\
\hline $45-49$ & 8 \\
\hline $50-54$ & 6 \\
\hline $55-59$ & 4 \\
\hline $60-64$ & 2 \\
\hline $65-69$ & 1 \\
\hline
\end{tabular}

Table 1. Age ranges of participants
This preliminary analysis will determine if there is a significant difference between predicted affect across two different driving scenarios. In the first scenario, participants are asked to predict their emotional state when driving a vehicle with a lane departure warning system. These systems are used to give drivers additional information about their position in the lane but do not increase the level of automation of the driving task. In the second scenario, participants are asked to forecast their emotional state when driving a vehicle with automated lane keeping, a system that automatically steers the vehicle for the driver if it is approaching the outside of a lane without an active turn signal.

The main hypotheses here are that there will be higher PA in the warning safety feature condition than the automated safety feature condition, and that there will be higher NA in the automated safety features condition. Driving confidence and technology experience will also be used to determine if there are high correlations between other factors that could effect these relationships.

\section{Methods}

Participants

A total of 195 participants (85 females, 109 males, and 1 individual who elected not to report gender) took part in the current study. The average age of participants was 33 years old $(S D=9.288)$, with a range and distribution as shown in Table 1. Participants were collected via Amazon Mechanical Turk and were paid $\$ 1.00$ (USD) for their participation in the survey, which lasted approximately 15 minutes.

\section{Materials}

Participants were asked a series of questions about their driving experiences, weekly time spent driving, and in what environment they believed they would use different automated and warning safety features. Participants were also asked about their experiences using technology by employing questions from the Selfdriving Car Acceptance Scale [5].

Items from the SCAS were rated on a seven point Likert scale from "Strongly disagree" to "Strongly agree":

- "I like to use technology to make tasks easier for me."

- "I have bad experiences when I try to use new technology instead of doing things 'the old fashioned way."

- "There are tasks in my life that have been made easier by computers doing the work for me."

Participants responded to four items discussing their confidence driving in different environments. They rated how much they agreed or disagreed with the statements on a seven point Likert scale ranging from "Strongly disagree" to "Strongly agree". Confidence items are listed below:

- I am confident in my driving abilities in urban environments.

- I am confident in my driving abilities in suburban environments.

- I am confident in my driving abilities in rural environments. 


\section{Vignettes}

Warning safety feature

"Imagine that you are driving a vehicle using a lane

departure warning system, a system that warns the driver when nearing the outside of a lane when the turn signal is not activated. To what degree would you experience each of the following feelings or emotions while driving this vehicle? Please respond to the scale below."

Automated safety feature "Imagine that you are driving a vehicle using automated lane keeping, a system that automatically steers the vehicle to maintain position within a lane. To what degree would you experience each of the following feelings or emotions while driving this vehicle? Please respond to the scale below."

Scale

- "I would feel interested." (positive affect)

- "I would feel distressed." (negative affect)
- I am confident in my driving abilities in highway environments.

Lastly, participants were asked to rate their forecasted positive and negative affect relating to two different vignettes. The first vignette asked participants to imagine themselves using a warning safety feature. The second vignette asked participants to imagine themselves using an automated safety feature.

\section{Results}

The positive and negative affect scores for each vignette were scored and averaged to get four ratings: average warning feature positive affect; average warning feature negative affect; average automated feature positive affect; and average automated feature negative affect. These scores were compared to determine if there was a significant difference between the different variables. Reliability tests were also conducted to construct validity with Cronbach's Alpha values of $\alpha=0.91, \alpha=0.92, \alpha=0.92$, and $\alpha=0.91$ respectively. These difference scores should show how much positive and negative affect differed within responses for the two vignettes, and how much positive and negative affect differed between vignettes.

The data, seen in Figure 1, showed that participants had significantly higher ratings of positive affect than negative affect in both the warning, $t(179)=9.31$, $p<0.001$, and automated feature vignettes, $t(181)=7.68, p<0.001$. Negative affect for automated safety features was significantly higher than warning safety features, $t(181)=2.29, p=0.023$. This shows that there was an overall more positive than negative emotion forecasted to be felt during both driving scenarios, regardless of level of automation. There was significantly more negative emotion forecasted to be experienced when using automated safety features than warning safety features.

A positive technology experience score was calculated based on the summed responses to three items from the SCAS. A reliability test was performed to determine construct validity, $\alpha=0.53$. This score is the amount of overall positive experiences reported by participants and is calculated by adding the results of the first and last items and subtracting the second item score. Higher scores indicate more positive experience with technology. Lower scores indicate less positive experience with technology.

Positive technology experience was positively correlated with average warning feature positive affect, $r(184)=0.25, p=0.001$, and average automated feature positive affect $r(187)=0.27, p<0.001$. Therefore, as positive technology experience increased, so did the forecasted positive emotions toward both warning and automated safety features. Positive technology experience was negatively correlated with average warning feature negative affect, $r(185)=-0.15$, $p=0.043$, and average automated feature negative affect, $r(187)=-1.97, p=0.006$. This shows that as positive technology experience decreases, negative affect increases toward both warning and automated safety features. In other words, those with more positive experiences with technology have higher levels of predicted positive emotions toward warning and safety features; whereas, those with less positive experiences toward technology have more negative emotions associated with both automated and warning safety features. 


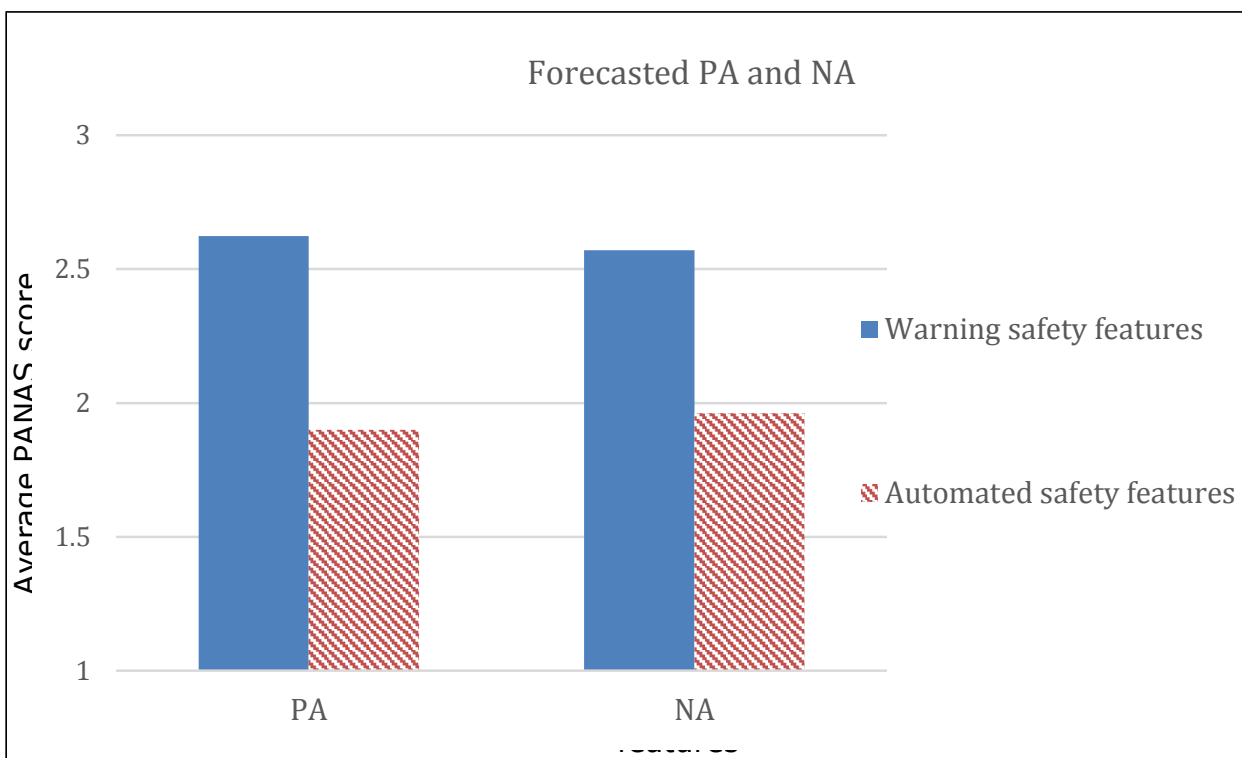

Figure 1. Results from comparison within and between automated and warning safety feature vignettes. significantly negatively correlated to negative affect predicted in the automated safety feature scenario, $r(186)=-0.16, p=0.032$. Therefore, as driving confidence increased, negative affect predicted to be felt toward automated safety features decreased. A similar effect was found toward warning safety features, as driving confidence increased, the predicted negative affect toward warning safety features decreased, $r(184)=-0.21, p=0.005$. No relationship was found between positive affect and overall driving confidence.

\section{Discussion}

Negative affect was found to be higher towards automated safety features than towards warning safety features. This could be due to correlational relationships with age, driving confidence, and experience with technology. Alternatively, it may be inflated due to the recent media attention of accidents related to automated vehicle systems.

Despite higher levels of negative affect towards automated safety features, participants had significantly higher positive than negative affect across both the automated and warning safety systems vignettes. This points toward an overall positive view of these systems. Higher positive affect was significantly correlated to positive technology experience for both warning and automated systems. Therefore, the experiences that people have with technology in general may influence their affective forecasting towards these systems.

Affective forecasting has been shown to be inaccurate in other settings such as student multitasking during homework completion [1]. Thus, further behavioral 
research will need to be conducted to determine whether the same is true in this context. Regardless, automotive manufacturers should be aware of the tendency to exaggerate predicted negative affect as this greatly influences perceptions of their vehicles.

Further exploration of the data collected in this study will help to determine the factors that predict perceived usefulness of automated and warning systems. Some of the factors that the authors are investigating are age, driving enjoyment, education, and driving environment. The findings from this research will help give insight into the minds of drivers to determine factors that influence vehicle automation acceptance and trust.

\section{Acknowledgements}

The authors would like to thank Dr. Michael A. Nees for his consultation in the present research. Portions of the work were supported by a National Science Foundation Graduate Research Fellowship (DGE-1148903).

\section{References}

1. Calderwood, C. Green, J. D., Joy-Gaba, J. A., Moloney, J. M. (2016). Forecasting errors in student media multitasking during homework completion. Computers \& Education 94, 2016. 3748

2. Dunn, E. W., \& Laham, S. M. (2006). Affective forecasting: a user's guide to emotional time travel. In J. P. Forgas (Ed.), Affect in social thinking and behavior (pp. 177-193). New York, NY: Psychology Press.

3. Endsley, M. \& Kaber, D. (1999). Level of automation effects on performance, situation awareness and workload in a dynamic control task. Ergonomics, 42, 462-492.
4. Gilbert, D. T., Pinel, E. C., Wilson, T. D., Blumberg, S. J., \& Wheatley, T. P. (1998). Immune neglect: a source of durability bias in affective forecasting. Journal of personality and Social Psychology, 75(3), 617-638.

5. Nees, M.A. (2016, September). Acceptance of Selfdriving Cars: An Examination of Idealized versus Realistic Portrayals with Self-driving Car Acceptance Scale. Proceedings of the HFES $60^{\text {th }}$ Annual Meeting, Washington, DC.

6. Rosenzweig, E. \& Critcher, C. R. (2014). Decomposing forecasting: the salienceassessment-weighting (SAW) model. Current Directions in Psychological Science, 23(5), 368373.

7. Sheridan, T. B. (1992). Telerobotics, Automation and Human Supervisory Control. Cambridge, MA: MIT Press.

8. Watson, D., Clark, L.A., \& Tellegen, A. 1988. Development and Validation of Brief Measures of Positive and Negative Affect: The PANAS Scales. Journal of Personality and Social Psychology 54, 6. 1063-1070 